\title{
Compression on trigger points in the leg muscle increases parasympathetic nervous activity based on heart rate variability
}

\author{
Kohichi Takamoto $\cdot$ Shigekazu Sakai $\cdot$ Etsuro Hori $\cdot$ \\ Susumu Urakawa · Katsumi Umeno · Taketoshi Ono · \\ Hisao Nishijo
}

Received: 9 December 2008/ Accepted: 27 January 2009/Published online: 21 February 2009

(C) The Author(s) 2009. This article is published with open access at Springerlink.com

\begin{abstract}
Massotherapy, the therapeutic use of massage, is used to treat various chronic pain syndromes. One type of massotherapy, pressure stimulus applied over trigger points (TPs), is reported to have excellent therapeutic effects. Its effect is possibly mediated through changes in the autonomic nervous system although little research has been conducted to assess autonomic activity during TP compression. We have investigated how compression applied over TPs affects the autonomic nervous system. Six healthy young adult females whose daily working routine was carried out predominantly in a standing position were enrolled in the study cohort. After a day's work, the subjects were asked to rest supine, and electrocardiograms (ECGs), instantaneous lung volume (ILV) and systolic and diastolic blood pressures (SBP, DBP) were measured before and after pressure application over the TPs in those lower limb muscles where the subjects felt muscle fatigue or discomfort. The subjects were also asked to coordinate breathing with the beeping sounds. The therapeutic effects of TP compression were assessed by a subjective fatigue scale. Parasympathetic nervous activity was also assessed by spectral analysis of heart rate (HR) variability. The transfer function from ILV to HR was evaluated using
\end{abstract}

K. Takamoto · S. Sakai · E. Hori · S. Urakawa - K. Umeno ·

T. Ono $\cdot$ H. Nishijo $(\square)$

System Emotional Science,

Graduate School of Medicine and Pharmaceutical Sciences,

University of Toyama, Sugitani 2630, Toyama 930-0194, Japan

e-mail: nishijo@med.u-toyama.ac.jp

K. Takamoto $\cdot$ S. Sakai $\cdot$ E. Hori - S. Urakawa - K. Umeno ·

T. Ono $\cdot$ H. Nishijo

Core Research for Evolutional Science and Technology

(CREST), Japan Science and Technology Agency (JST),

Kawaguchi, Japan linear analysis. The results indicated that TP compression (1) decreased HR, SBP and DBP, (2) increased parasympathetic activity, (3) increased the gain from ILV to HR, and (4) improved the fatigue scores. These findings suggest that an increase in parasympathetic nervous activity after the TP compression induced a reduction of fatigue. The therapeutic mechanisms of TP compression to enhance parasympathetic nervous system are discussed.

Keywords Autonomic nervous system - Heart rate . Massage $\cdot$ Respiration $\cdot$ Transfer function

\section{Introduction}

Recent reviews on clinical pain have highlighted the difference between different pain etiologies and endogenous pain-inhibiting mechanisms [1,2]. Abnormalities of autonomic functions have been implicated in chronic pain in certain diseases. For example, sympathetic overactivation and/or parasympathetic hypoactivation were reported in fibromyalgia with diffuse tenderness and musculoskeletal pain [3], in reflex sympathetic dystrophy and causalgia [4], and in whiplash-associated disorders [5]. Such abnormalities in the autonomic nervous system may generate and sustain chronic pain [5-7].

Massage, including muscle compression, has been used for pain relief since ancient times and still remains in use today [8]. Recent review articles suggest that massage therapy may be beneficial in managing various subacute and chronic pain conditions $[9,10]$. In massage therapy, mechanical stimulus by touch and manipulation with the hands of the therapist, such as pressing, rubbing, and stroking, can have therapeutic effects, including pain relief, improved local circulation, and the release of muscle strain 
$[11,12]$. In therapeutic massage for pain relief, spots with focal tenderness in a taut band of muscle are called trigger points (TPs). These are painful and can evoke a characteristic referred pain in areas of the body far away from their actual location, a flexion withdrawal reflex (jump sign), and local muscle contraction (twitch response) on compression [13, 14]. Pressure application over TPs has been reported to be particularly beneficial in the massotherapy for myofascial pain syndrome [15-17].

Melzack et al. [18] reported a remarkably high degree (71\%) of correspondence between TPs and acupuncture points. Qualified practitioners of acupuncture therapy know that acupressure or an acupuncture stimulus applied onto TPs activates autonomic nervous reactions, such as vasoconstriction, vasodilation, cold sweat, perspiration, and decrease or increase of secretion [14]. The results of an earlier study by our group indicated that placing acupuncture needles into TPs increased parasympathetic nervous activity [19]. Although these findings suggest that the therapeutic effect of massotherapy with TP stimulation is associated with changes in the autonomic nervous system, details of the effects on the autonomic nervous system are still unknown. We have therefore have studied how autonomic nervous activity, in particular the activity of the parasympathetic nervous system, is affected by compression applied to the TPs in the lower-limb muscle, using spectral analysis of heart rate (HR) variability as an indicator.

In the spectral analysis of HR variability, the longitudinal data of RR intervals per unit time is first subjected to frequency analysis by means of fast Fourier transform (FFT), maximum entropy method (MEM), or the autoregressive method (AR), and the extracted spectral component in HR variability is then used for autonomic function assessment. The reliability of spectral data in assessing autonomic status has been reported: the highfrequency (HF) component of HR variability (HRV) reflects activity of the vagal parasympathetic system, and its low-frequency (LF) component includes both sympathetic and parasympathetic activities [20,21].

It is noted that the HF component of HR variability, which was the main target of this study, has been reported to be dependent on the breathing cycle and to be affected by breathing rate and tidal volume [22-25]. Only one earlier study has investigated the effects of TP massage on autonomic nervous activity and reported an increase in parasympathetic nervous activity based on spectral analysis of HR variability [17]. However, the study did not analyze the effects of respiration on HR. It has also been reported that the transfer gain above $0.15 \mathrm{~Hz}$ from the instantaneous lung volume (ILV) to the HR is mediated mainly through parasympathetic nervous activity [26]. To elucidate the influence of breathing on the HR before and after pressure application over TPs, we also analyzed the transfer function from ILV to the HR by asking the subjects to breathe at random cycles for broader respiration-induced fluctuations of HR variability [27]. Thus, we investigated how TP massage to the lower limb muscles modulated HR variability.

\section{Methods}

\section{Subjects}

Six female subjects (average age $21.0 \pm 0.0$, mean \pm SEM) whose daily working routine was carried out predominantly in a standing position (8:30 a.m. to 4:00 p.m) were enrolled in this study. All were non-smokers, and none had any medical problems known to affect HR variation. All subjects were treated in strict compliance with the Declaration of Helsinki and the U.S. Code of Federal Regulations for the protection of human subjects. The experiments were conducted with the understanding and consent of each subject, and they had received the approval of the ethical committee of our university.

\section{Pressure on TPs}

Pressure was manually applied over the TPs in the lowerlimb muscles where the subjects felt fatigue and discomfort after many hours of standing. In order to maintain consistency, the same therapist (S.S.), a fellow of the National Council of Judo Therapists, performed all massage treatments. The massage procedure, which took 20 min each time, employed the techniques described by Travell and Simons [14]. The authors called this technique "ischemic compression". The intensity of pressure was controlled to a level at which each subject reported "comfortable pain".

\section{Recording of autonomic functions}

Autonomic functions were measured before and after compression of the TPs. The left arm was kept at the level of the right atrium, and a noninvasive tonometric blood pressure (BP) transducer, connected to a $\mathrm{BP}$ monitor (Jentow 7700; Colin, Japan), was strapped over the left radial artery. The sphygmomanometer cuff of an oscillometric BP recording device for calibration of the tonometric transducer was attached to the upper left arm. Surface electrodes were attached to the chest for recording of the electrocardiogram (ECG). The facemask was placed on the subject's face, and air flow (ILV) was measured by a respiration monitor (RM200; Minato, Japan). 
These autonomic data, including trigger signals synchronized with the QRS-waves of the ECGs, ILV, and BP, were digitized using an A/D converter with a sampling rate of $1 \mathrm{kHz} / \mathrm{ch}$ and recorded on a hard disk. Both systolic and diastolic BP (SBP, DBP) was measured from the digitized continuous BP data.

\section{Study protocol}

Measurements were performed in a temperature-controlled room $\left(26^{\circ} \mathrm{C}\right)$. After standing for most of the day, the subjects were asked to rest supine in a quiet $3 \times 3 \times 5-\mathrm{m}$ room equipped with an exhaust fan to re-ventilate the room air continuously. The electrodes for the ECGs and probes for the BP and IVL were then placed at the appropriate positions (see above).

After the various electrodes and probes has been made ready, the TPs were identified by the therapist. The subjects were then allowed to rest in a supine position for $20 \mathrm{~min}$ before recording. Results from earlier studies showed that, compared with the supine position, the upright position itself changes both blood distribution in the body due to gravity and HR variability. The upright position may increase sympathetic activity and mask the effects of TP compression. Therefore, the subjects were placed in the supine position in our study, in accordance with most of the previous studies on HR variability. After recording the control data in the supine position for $20 \mathrm{~min}$, compression was applied on the TPs, and the data recorded for $20 \mathrm{~min}$ after the massage. These two sets data (each for $20 \mathrm{~min}$ ) were subjected to spectral analyses. During the measurements, the subjects were instructed to coordinate breathing with the beeping sounds generated at random time intervals in the 0.12- to $0.4-\mathrm{Hz}$ band (random frequency breathing). No special instruction on breathing depth was given to the subjects, and the subjects were allowed to breath at their own natural depth. To determine the influence of random frequency breathing on HR variability and BP fluctuation, data were also taken from the subjects during constant frequency breathing $(0.25 \mathrm{~Hz})$.

The subjective sense of fatigue was assessed before and after compression on the TPs. In a questionnaire, each subject was asked to evaluate her fatigue level subjectively on a 5-point scale where a score of 5 indicated "very fine", 4 indicated "fine", 3 indicated "OK (not tired)", 2 indicated "tired", and 1 indicated "exhausted".

\section{Data analysis}

After removing the electrical noise artifact, the data were analyzed using a spectral technique by applying spline interpolation at $1.0 \mathrm{~Hz}$. For example, the raw data of the RR intervals of the ECGs is a discontinuous function of the time interval between two consecutive $\mathrm{R}$ waves. This discontinuous function is transformed into a continuous function by means of interpolation and then subjected to re-sampling at mean RR intervals to generate sampling data at equal time intervals. For the spectral analysis, the MEM was used. According to the protocol by Akselrod et al. [20], the sum of the spectral power obtained by MEM within $0.05-0.15 \mathrm{~Hz}$ was used as the LF, whereas the sum of power within 0.15 $0.50 \mathrm{~Hz}$ was used as the HF [20, 28]. The transfer function from ILV to HR was assessed by means of linear analysis, and the mean coherence and gain were computed.

All data were expressed as the mean \pm standard error of the mean (SEM). Statistical analyses were performed by a paired $t$ test. A value of $P<0.05$ was defined as being statistically significant.

\section{Results}

Effects on cardiovascular functions

After application of a pressure stimulus over the TPs during random breathing, the mean RR interval of the six subjects increased significantly from 920 to $950 \mathrm{~ms}$ (Fig. 1a), and the HR decreased significantly from 67.3 to $65.2 \mathrm{bpm}$ (paired $t$ test, $P<0.05$ ). The mean SBP and DBP of the six subjects decreased significantly from 119 to $102 \mathrm{mmHg}$, and from 68 to $54 \mathrm{mmHg}$, respectively (paired $t$ test, $P<0.05$ ) (Fig. 1b). The results were essentially the same under constant frequency breathing $(0.25 \mathrm{~Hz})$. No significant difference was detected between these values measured during random breathing and those measured during constant frequency breathing $(0.25 \mathrm{~Hz})$ (Fig. 1a, b) (paired $t$ test, $P>0.05$ ).

\section{Effects on autonomic activity}

Figure 2 shows an example of the spectral analysis of HR variability during random breathing. Pressure application over the lower-limb muscle of a subject increased both the LF and HF components. Figure $3 \mathrm{a}$ shows the power spectral density (PSD) of the HF component of individual subjects, which was measured before and after the pressure application over the TPs during random breathing. An increase in PSD was observed in all subjects after pressure application, with the increase ranging from 5 to $125 \%$. The mean of the HF component of all subjects also showed a significant increase after pressure application over the TPs under both random and constant frequency breathing conditions (Fig. 3b) (paired $t$ test, $P<0.05$ ). No significant difference was detected between the values measured during random breathing and those measured during constant frequency breathing (paired $t$ test, $P>0.05$ ). 

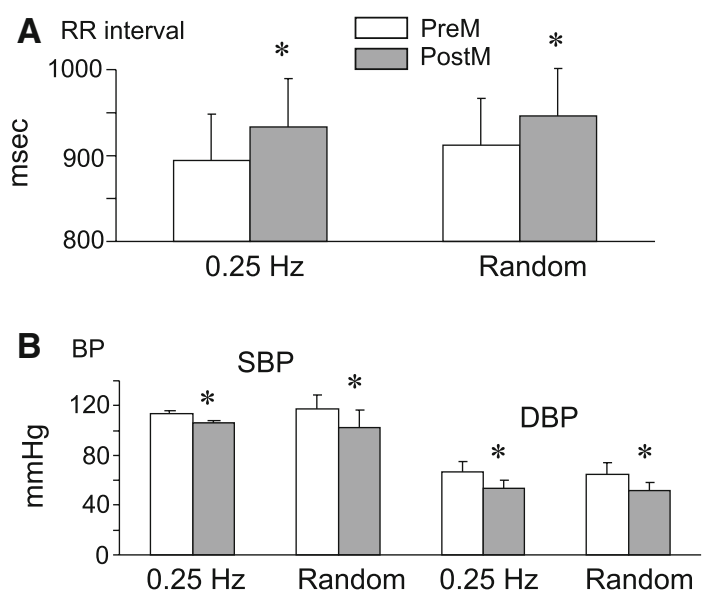

Fig. 1 Cardiovascular changes after the application of a pressure stimulus over trigger points (TPS) in the lower-limb muscle. a Changes in mean RR interval on the electrocardiograms $(n=6)$. b Changes in mean systolic and diastolic blood pressure (SBP and $D B P$, respectively; $n=6$ ). A significant increase in mean RR intervals and a significant decrease in mean SBP and DBP were observed after the application of pressure stimulus during constant and random frequency breathing. No difference was observed between the values measured during constant and random frequency breathing. PreM Before pressure application over TPs, PostM after pressure application over TPs, $0.25 \mathrm{~Hz}$ constant frequency breathing at $0.25 \mathrm{~Hz}$, random random frequency breathing. $* P<0.05$

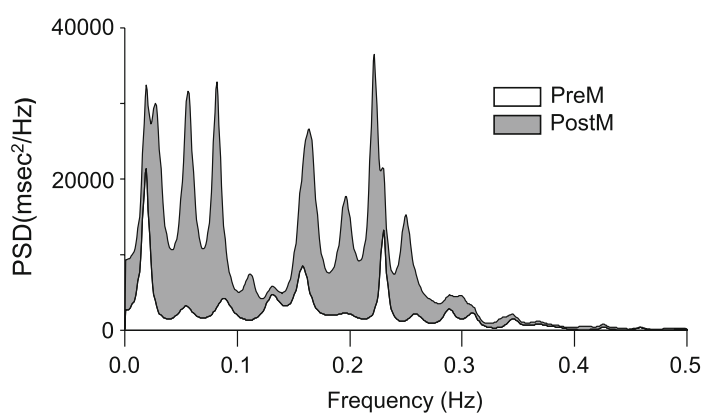

Fig. 2 An example of a spectral analysis of the RR interval. Lowfrequency $(L F)$ and high-frequency $(H F)$ components increased after pressure application over the TPs. Vertical scale Power spectral density (PSD), horizontal scale frequency (LF component 0.05$0.15 \mathrm{~Hz}$; HF component $0.15-0.50 \mathrm{~Hz}$ ). See caption to Fig. 1 for explanation of abbreviations

The coherence between ILV and HR during random breathing was relatively high (within a range from 0.4 to 0.8 ) regardless of whether or not the pressure was applied over the TPs (Fig. 4). This high coherence indicates the statistical validity of the transfer function analysis. A comparison of the mean gain in HF band $(0.15-0.50 \mathrm{~Hz})$ showed a significant increase after pressure application over the TPs under random frequency breathing (Fig. 5) (paired $t$ test, $P<0.05$ ). No significant difference was
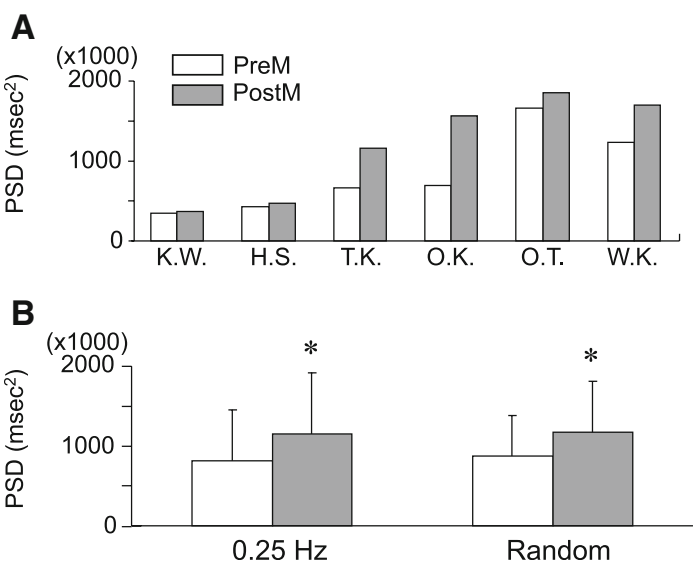

Fig. 3 Changes in the HF component of heart rate $(H R)$ variability after pressure application over TPs in the lower-limb muscle. a Changes in the HF component in each subject $(n=6)$. Initials of each subject appear under the horizontal scale. b Changes in the mean HF component $(n=6)$. A significant increase in the mean HF component was observed after the application of pressure stimulus during constant and random frequency breathing. No difference was observed between the values measured during constant and random frequency breathing. See caption to Fig. 1 for explanation of abbreviations

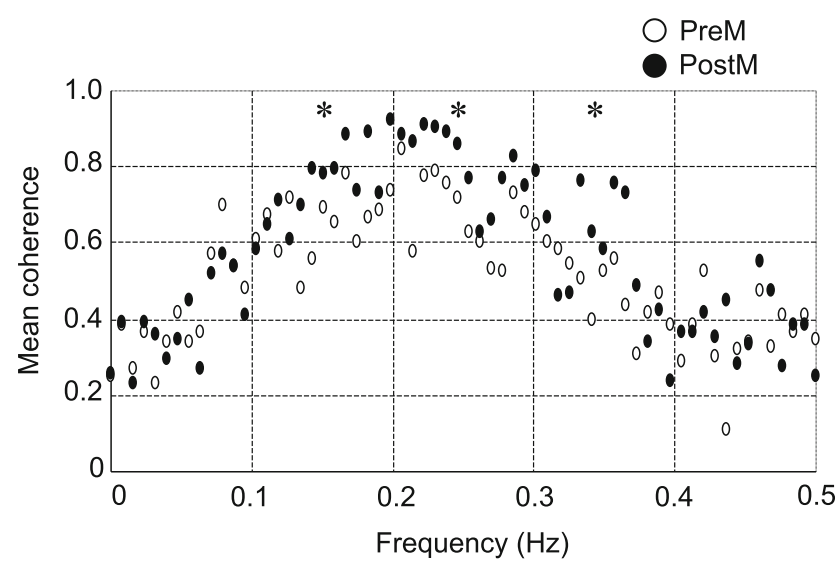

Fig. 4 Change in mean coherence during random frequency breathing after the application of pressure over the TPs in the lower-limb muscle. Changes in mean coherence between instantaneous lung volume $(I L V)$ and HR after the application of pressure over TPs is shown. The vertical and horizontal scales represent the mean coherence between ILV and HR $(n=6)$ and breathing frequency $(\mathrm{Hz})$, respectively. The mean coherence both before and after pressure application indicated a strong correlation in the HF breathing zone $(0.15-0.50 \mathrm{~Hz})$. Asterisk indicates significant difference from the control (preM) $(P<0.05)$

detected between the gains measured during random breathing and those measured during constant frequency breathing (paired $t$ test, $P>0.05$ ). These findings indicate that the influence of respiration on the HR increased in the HF range after pressure had been applied on the TPs. 


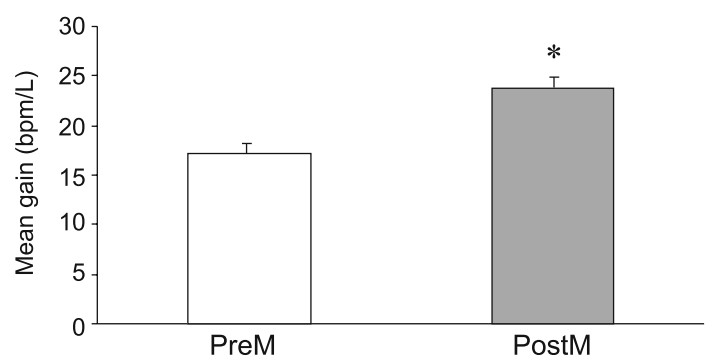

Fig. 5 Changes in mean gain of the transfer function during random frequency breathing after the application of pressure over the TPs points in the lower-limb muscle. Changes in the mean transfer magnitude between ILV and HR after the application of pressure onto TPs is shown $(n=6)$. A significant increase in the mean transfer magnitude was observed after pressure application over TPs. Asterisk indicates significant difference from the control ( preM $)(P<0.05)$

Table 1 Changes in subjective fatigue scores before and after pressure application over trigger points

Subject K.W. H.S. T.K. O.K. O.T. W.K. Mean \pm SEM (initials)

\begin{tabular}{llllllll}
\hline PreM & 3 & 1 & 1 & 2 & 2 & 2 & $1.83 \pm 0.75$ \\
PostM & 4 & 4 & 4 & 5 & 4 & 4 & $4.17 \pm 0.41$ \\
\hline
\end{tabular}

PreM, Before pressure application over TPs; PostM, after pressure application onto TPs; SEM, standard error of the mean

Five-point scale: 5, "very fine"; 4, "fine"; 3, "OK (not tired)"; 2, "tired"; 1, "exhausted"

Changes in the sense of fatigue

Due to long hours of standing each and every working day, each subject had strong feeling of congestion, fatigue, and discomfort in the lower extremities before the pressure stimulus was applied onto the TPs. The mean subjective fatigue score showed a significant improvement following pressure application: from 1.83 to 4.17 (Signed rank sum test, $P<0.05)$ (Table 1).

\section{Discussion}

Effect of pressure application over TPs on autonomic activity

The HF component of HR variability increased with a concomitant decrease in SBP and DBP following the application of pressure over the TPs in the lower-limb muscle. These findings suggest that relative activity of parasympathetic nervous system was increased. However, the HF component of HR variability has been reported to be dependent on the respiration cycle and affected by respiration frequency and tidal volume [22-25]. Burst firing of the parasympathetic nerve (vagal nerve) disappears or decreases during inspiration, and the HR variability associated with the disappearance or decrease of the burst firing is observed as the HF component (i.e., respiratory sinus arrhythmia). Thus, the HF component of HR variability is dependent on breathing, and the influence of respiration on HR variability is considered to be an index of the influence of parasympathetic activity on HR variability [29-31]. Therefore, we analyzed a transfer function between ILV and HR.

A previous study on phase and gain in the ILV-HR transfer function reported that this transfer function had characteristics of a first-order low-pass filter [26, 29]. In our study, the analysis of ILV to HR transfer function displayed a high coherence, ranging from 0.4 to 0.8 between ILV and HR; this high coherence indicates the validity of this analysis. The results indicate that the mean gain between ILV and HR increased significantly after the application of pressure over TPs under both random and constant frequency breathing. These findings suggest that the increase in the HF component after TP compression can be attributed to an increase in the influence of respiration on HR variability. It should also be noted that the increase in the gain was confined to the HF band $(0.15-0.5 \mathrm{~Hz})$ in the present study. Consistent with the present study, a previous study reported that the transfer gain above $0.15 \mathrm{~Hz}$ was mediated mainly through parasympathetic nervous activity [26]. These findings strongly suggest that compression over TPs is capable of activating the parasympathetic nerve system.

\section{Physiological effects of TP compression}

Previous studies have reported the induction of sympathetic hyperactivity in various pathophysiological conditions, such as the accumulation of metabolic byproducts and production of inflammatory substance due to ischemia in the forearm, congestion in the lower extremities due to negative pressure, and reduced cardiac output caused by diminished venous return due to congestion [32-34]. Our results, together with these of the earlier studies, suggest that both the relief of pain induced by the perfusion of metabolic byproducts and inflammatory substance due to TP compression and the increase in cardiac output due to decreased congestion induced by TP compression may result from a suppression of sympathetic nervous activity and an increase in parasympathetic nervous activity. Since an increase in parasympathetic nervous activity itself increases peripheral blood flow [35] and is one of the important factors to relief chronic pain in the muscle [19, 35-37], the increase in parasympathetic nervous activity induced by the above peripheral mechanisms may further decrease chronic pain or muscle fatigue. 
However, we did not directly analyze sympathetic activity in our study since the LF component of the HR variability reflects both sympathetic and parasympathetic activity [21], and the LF/HF ratio represents the balance between sympathetic and parasympathetic nervous systems $[38,39]$. Further studies will be required to directly monitor muscle sympathetic nerve activity to assess the therapeutic effect of pressure application over TPs.

\section{Therapeutic effects of TP compression}

Our analysis of the subjective fatigue score indicates that the score was significantly improved after TP compression. A decrease in parasympathetic nervous activity is one of the important characteristics of fibromyalgia and chronic fatigue syndrome [40, 41]. Compression of TPs induces various physiological changes in the lower extremities, resulting in an increase in parasympathetic nervous activity, as discussed above. The autonomic afferent fibers may detect these changes and directly affect the limbic system, which is involved in emotion and circulation control. This central mechanism may also contribute to changes in the fatigue score. Furthermore, subjective fatigue sensation is known to be significantly related to muscle sympathetic nervous activity [42]. Therefore, the increase in parasympathetic nervous activity may decrease muscle sympathetic nervous activity, which results in the decease in fatigue sensation. These findings on an increase in parasympathetic nervous activity suggest that TP compression released the subjects from the stress arising from working in a standing position over a long period.

In the study reported here, we carried out an analysis of TPs, which are reported to have a high therapeutic potential for pain management $[14,16,17]$. In future studies, the effects of compression on regions other than TPs should be assessed to investigate the specific characteristics of the TPs.

\section{Conclusions}

The results of our study demonstrate that pressure application over TPs in the lower-limb muscles induced various physiological effects, such as (1) increased RR intervals (decreased HR) and decreased SBP and DBP, (2) increased HF component in HR variability, and (3) increased gain of the transfer function from ILV to HR in the HF band. These findings suggest that TP compression can elevate the activity of the parasympathetic nervous system and provide some insight into the physiological mechanisms of pain relief induced by the pressure application over TPs.
Open Access This article is distributed under the terms of the Creative Commons Attribution Noncommercial License which permits any noncommercial use, distribution, and reproduction in any medium, provided the original author(s) and source are credited.

\section{References}

1. Woolf CJ (2004) Dissecting out mechanisms responsible for peripheral neuropathic pain: implications for diagnosis and therapy. Life Sci 74:2605-2610. doi:10.1016/j.lfs.2004.01.003

2. Woolf CJ, American College of Physicians, American Physiological Society (2004) Pain: moving from symptom control toward mechanism-specific pharmacologic management. Ann Intern Med 140:441-451

3. Furlan R, Colombo S, Perego F, Atzeni F, Diana A, Barbic F, Porta A, Pace F, Malliani A, Sarzi-Puttini P (2005) Abnormalities of cardiovascular neural control and reduced orthostatic tolerance in patients with primary fibromyalgia. J Rheumatol 32:17871793

4. Baron RM, Levine JDMP, Fields HMP (1999) Causalgia and reflex sympathetic dystrophy: does the sympathetic nervous system contribute to the generation of pain? Muscle Nerve 22:678-695. doi:10.1002/(SICI)1097-4598(199906)22:6<678:: AID-MUS4>3.0.CO;2-P

5. Passatore M, Roatta S (2006) Influence of sympathetic nervous system on sensorimotor function: whiplash associated disorders (WAD) as a model. Eur J Appl Physiol 98:423-449. doi:10.1007/ s00421-006-0312-8

6. Janig W (1992) Pain and the sympathetic nervous system: pathophysilogical mechanism. In: Bannister SR, Mathias CJ (eds) Autonomic failure, 3rd edn. Oxford University Press, Oxford, pp 231-251

7. Schott GD (1999) Pain and the sympathetic nervous system. In: Mathias CJ, Bannister SR (eds) Autonomic failure, 4th edn. Oxford University Press, Oxford, pp 520-526

8. Schiotz EH (1958) Manipulation therapy of the spinal column from the viewpoint of medical history. Tidsskr Nor Laegeforen 78:946-950

9. Furlan AD, Brosseau L, Imamura M, Irvin E (2002) Massage for low-back pain: a systematic review within the framework of the Cochrane collaboration back review group. Spine 27:1896-1910. doi:10.1097/00007632-200209010-00017

10. Tsao JSCI (2007) Effectiveness of massage therapy for chronic, non-malignant pain: a review. Evidence-based complementary and alternative medicine 4:165-179. doi:10.1093/ecam/nel109

11. Cafarelli E, Flint F (1992) The role of massage in preparation for and recovery from exercise. Sports Med 14:1-9. doi:10.2165/ 00007256-199214010-00001

12. Mori H, Ohsawa H, Tanaka TH (2004) Effect of massage on blood flow and muscle fatigue following isometric lumbar exercise. Med Sci Monit 10:CR173-178

13. Travell J, Rinzler S (1952) The myofascial genesis of pain. Postgrad Med 11:425-434

14. Travell J, Simons DG (1983) Myofascial pain and dysfunction: the trigger point manual. Williams \& Wilkins, Baltimore

15. Simons DG, Travell JG (1983) Myofascial origins of low back pain. 1. Principles of diagnosis and treatment. Postgrad Med 73: 66, 68-70, 73

16. Simons DG (1984) Myofascial pain syndromes. Arch Phys Med Rehabil 65(9):561

17. Delaney JPA, Leong KS, Watkins A, Brodie D (2002) The shortterm effects of myofascial trigger point massage therapy on cardiac autonomic tone in healthy subjects. J Adv Nurs 37:364371. doi:10.1046/j.1365-2648.2002.02103.x 
18. Melzack R, Stillwell DM, Fox EJ (1977) Trigger points and acupuncture points for pain: correlations and implications. Pain 3:3-23. doi:10.1016/0304-3959(77)90032-X

19. Sakai S, Umeno K, Hori E, Kitabayashi N, Ono T, Nishijo H (2007) Specific acupuncture sensation correlates with EEGs and autonomic changes in human subjects. Autonomic Neurosci Basic Clin 133:158-169. doi:10.1016/j.autneu.2007.01.001

20. Akselrod S, Gordon D (1981) Power spectrum analysis of heart rate fluctuation: a quantitative probe of beat-to-beat cardiovascular control. Science 213:220-222. doi:10.1126/science.6166045

21. Pomeranz B, Macaulay JB, Margaret A (1985) Assessment of autonomic function in humans by heart rate spectral analysis. Am J Physiol 248(1 Pt 2):H151-H153

22. Katona PG, Poitras JW, Barnett GO (1970) Cardiac vagal efferent activity and heart period in the carotid sinus reflex. Am J Physiol 218:1030-1037

23. Hirsch JA, Beverly B (1981) Respiratory sinus arrhythmia in humans: how breathing pattern modulates heart rate. Am J Physiol 241:H620-H629

24. Grossman P, Karemaker J, Wieling W (1991) Prediction of tonic parasympathetic cardiac control using respiratory sinus arrhythmia: the need for respiratory control. Psychophysiology 28:201216. doi:10.1111/j.1469-8986.1991.tb00412.x

25. Grossman P, Kollai M (1993) Respiratory sinus arrhythmia, cardiac vagal tone, and respiration: within- and between-individual relations. Psychophysiology 30:486-495. doi:10.1111/j.14698986.1993.tb02072.x

26. Saul JP, Begger RD, Albrecht P, Stein SP, Chen MH, Cohen RJ (1991) Transfer function analysis of the circulation: unique insights into cardiovascular regulation. Am J Physiol 261:H1231-1245

27. Berger RD, Saul JP, Cohen RJ (1989) Assessment of autonomic response by broad-band respiration. IEEE Trans Biomed Eng 36:1061-1065. doi:10.1109/10.40812

28. Dishman RK, Nakamura Y, Garcia ME, Thompson RW, Dunn AL, Blair SN (2000) Heart rate variability, trait anxiety, and perceived stress among physically fit men and women. Int $\mathbf{J}$ Psychophysiol 37:121-133. doi:10.1016/S0167-8760(00)00085-4

29. Saul JP, Begger RD, Chen MH (1989) Transfer function analysis of autonomic regulation II. respiratory sinus arrhythmia. Am J Physiol 256(1 Pt 2):153-161

30. Taha BH, Simon PM, Dempsey JA, Skatrud JB, Iber C (1995) Respiratory sinus arrhythmia in humans: an obligatory role for vagal feedback from the lungs. J Appl Physiol 78:638-645
31. Yana K, Saul JP, Berger RD (1993) A time domain approach for the fluctuation analysis of heart rate related to instantaneous lung volume. IEEE Trans Biomed 40:74-81

32. Sanders JS, Ferguson DW (1988) Cardiopulmonary baroreflexes fail to modulate sympathetic responses during isometric exercise in humans: direct evidence from microneurographic studies. J Am Coll Cardiol 12:1241-1251

33. Scherrer U, Vissing SF, Victor RG (1988) Effects of lower-body negative pressure on sympathetic nerve responses to static exercise in humans. Circulation 78:49-59

34. Ray CA, Secher NH, Mark AL (1994) Modulation of sympathetic nerve activity during posthandgrip muscle ischemia in humans. Am J Physiol 266(1 Pt 2):H79-83

35. Ishii H, Niioka T, Watanabe H, Izumi H (2007) Inhibitory effects of excess sympathetic activity on parasympathetic vasodilation in the rat masseter muscle. Am J Physiol 293:R729-R736. doi: 10.1152/ajpcell.00018.2007

36. Cottingham JT, Porges SW, Lyon T (1988) Effects of soft tissue mobilization (Rolfing pelvic lift) on parasympathetic tone in two age groups. Phys Ther 68:352-356

37. Cottingham JT, Porges SW, Richmond K (1988) Shifts in pelvic inclination angle and parasympathetic tone produced by Rolfing soft tissue manipulation. Phys Ther 68:1364-1370

38. Pagani M, Lombardi F, Guzzetti S (1986) Power spectral analysis of heart rate and arterial pressure variabilities as a marker of sympatho-vagal interaction in man and conscious dog. Circ Res 59:178-193

39. Montano N, Ruscone TG, Porta A, Lombardi F, Pagani M, Malliani A (1994) Power spectrum analysis of heart rate variability to assess the changes in sympathovagal balance during graded orthostatic tilt. Circulation 90:1826-1831

40. Cordero DL, Sisto SA, Tapp WN, LaManca JJ, Pareja JG, Natelson BH (1996) Decreased vagal power during treadmill walking in patients with chronic fatigue syndrome. Clin Auton Res 6:329-333. doi:10.1007/BF02556303

41. Buskila D (2001) Fibromyalgia, chronic fatigue syndrome, and myofascial pain syndrome. Curr Opin Rheumatol 13:117-127. doi:10.1097/00002281-200103000-00005

42. Saito M, Mano T, Iwase S (1989) Sympathetic nerve activity related to local fatigue sensation during static contraction. J Appl Physiol 67:980-984 